Voix et Images

\title{
Du privé au politique : la Constellation du Cygne de Yolande Villemaire
}

\section{Suzanne Lamy}

Volume 13, numéro 1 (37), automne 1987

Suzanne Lamy

URI : https://id.erudit.org/iderudit/200680ar

DOI : https://doi.org/10.7202/200680ar

Aller au sommaire du numéro

Éditeur(s)

Université du Québec à Montréal

ISSN

0318-9201 (imprimé)

1705-933X (numérique)

Découvrir la revue

Citer cet article

Lamy, S. (1987). Du privé au politique : la Constellation du Cygne de Yolande

Villemaire. Voix et Images, 13(1), 18-28. https://doi.org/10.7202/200680ar d'utilisation que vous pouvez consulter en ligne.

https://apropos.erudit.org/fr/usagers/politique-dutilisation/ 


\title{
Du privé au politique: la Constellation $d u$ Cygne de Yolande Villemaire
}

\author{
par Suzanne Lamy $\dagger$
}

Pourquoi ce texte sur Yolande Villemaire, écrivaine peu connue aux ÉtatsUnis mais qui, au Québec, jouit d'une reconnaissance certaine? Pour plusieurs raisons. D'une part, il est bon que l'on sorte des sentiers battus et rebattus et que des écrivains autres qu'Anne Hébert ou Yves Thériault franchissent la frontière. D'autre part, suivre le courant des consécrations rapides comme il s'en fait au Québec peut être un bon sujet de réflexion. Le cas de Yolande Villemaire nous paraît illustrer à merveille cet effet d'entraînement par la réception qu'a eue son dernier roman. De plus, cet ouvrage, la Constellation du Cygne nous intéresse sur le plan théorique: il nous donne l'occasion de réfléchir sur le retour en force du référent, ce qui doit être pris en compte par la critique actuelle. Il n'est plus question de le tenir à distance. Et même l'idéologie peut à son tour être questionnée.

Tout d'abord qui est Yolande Villemaire? (Ce qui suit n'est pas exhaustif le moindrement mais vise seulement à situer Yolande Villemaire dans le milieu culturel québécois).

C'est une jeune écrivaine de moins de 40 ans, qui a présenté des performances, qui a commencé une thèse de doctorat sur Michel Tremblay. Elle s'est d'abord inscrite dans le courant de la contre-culture par sa collaboration à Hobo-Québec, elle s'est tournée vers les États-Unis, la vie urbaine, mais aussi vers l'Égypte et les sciences occultes. Elle a écrit plusieurs recueiłs de poèmes, aux titres originaux: Machine-t-elle (1974), Que stage blood (1977). Son roman, de facture très libre, la Vie en prose en 1981, a eu du succès auprès des intellectuels et lui a valu le prix des jeunes écrivains du Journal de Montréal. Dans Adrénaline, Yolande Villemaire a réuni des textes de poésie et prose, s'échelonnant de 1973 à 1982. Son roman, la Constellation du Cygne (1985), lui a mérité le prix du Journal de Montréal. Yolande Villemaire a aussi créé une spirale d'écrivantes du nom de Rrose Sélavy en 1984. En 1985, paraissait Quartz et mica, un recueil de poésie.

Deux prix, plus un dossier, dans la revue de l'Uqam Voix \& images au printemps 86, témoignent donc de la consécration dont a fait l'objet Yolande Villemaire. 
Or, ce livre, la Constellation du Cygne, je l'ai reçu comme un lacis d'interrogations, sur le roman lui-même et sur la lecture qui en a été faite. Plus que cela, ce texte publié aux éditions de la Pleine Lune (éditions de femmes) oblige à faire retour sur l'écrivaine elle-même par la longue entrevue accordée à Lucie Robert et parue dans le même numéro de Voix \& images.

D'emblée la Constellation du Cygne m'est apparu comme un excellent exemple pour aborder la question du privé et du politique puisque l'intrigue tient dans la relation amoureuse entre une prostituée juive et un officier nazi, relation qui débute le 15 août 1940 à Paris - jour de l'Assomption - et se termine en août 1944, s'étend donc sur les quatre années cruciales de la demière guerre.

Noeud d'interrogations que ce roman. Pour moi. Pour d'autres aussi. Pour les Juifs en particulier. Et aussi pour ceux et celles qui sont préoccupé(e)s par le politique. Le malaise que peut produire ce roman, il est mis en évidence dans l'éditorial signé par Bemard Andrès, directeur de la revue Voix \& images et qui donne comme titre à son intervention: «Du littéraire et du spirituel».

Ce n'est donc pas d'une lecture qui ne serait que personnelle qu'a germé ce texte, mais aussi de la distance existant entre le commentaire de Bernard Andrès d'une part, et le roman et les paroles échangées au cours de l'entrevue entre Yolande Villemaire et Lucie Robert d'autre part.

\section{Bernard Andrès écrit:}

Sans tomber dans la dichotomie facile (matérialisme historique versus syncrétisme débridé), on ne peut que constater l'abîme entre une réflexion théorique souvent austère, doublée d'une conception militante de la littérature et la capricieuse dynamique du propos villemairien, ressourcé à tous les avatars de l'orientalisme, s'appropriant avec une aisance déconcertante la symbolique du tarot, les merveilles de l'hologramme et l'enfer de l'holocauste. Non sans un certain malaise parfois sensible dans l'entrevue. (...)

Mais le problème avec ces débordements de l'imaginaire, c'est qu'ils charrient parfois le meilleur et le pire et sous le louable prétexte de rompre avec toute hiérarchisation, ils en viennent, pour la baliser, à banaliser l'Histoire (l'histoire culturelle, l'histoire politique). (...) Nul doute qu'en faisant flèche de toute croix (y compris la gammée), l'écriture désamorce alors l'Histoire 1.

La citation est longue, mais elle situe de façon tout à fait pertinente le problème posé par ce texte, soit le rapport à l'Histoire, à l'histoire culturelle et à l'histoire politique.

1 Bernard Andrès, «Du littéraire et du spirituel», Volx \& images, no 33, printemps 1986, p. $380-381$. 
Je tenterai de montrer combien cette relation me paraît pernicieuse en m'arrêtant sur: amour2;

- la forte intertextualité du texte avec celui de Duras: Hiroshima mon

- l'écriture prétendument réaliste du roman (en montrant comment elle se donne pour réaliste sur certain point [celui des scènes érotiques]); en fait combien tout cela s'inscrit dans une tradition littéraire et combien l'ensemble est tissé d'invraisemblances;

- enfin, à partir de l'amalgame pratiqué ici qui fond l'Histoire dans le grand Tout des alchimistes, je parlerai du choix à faire entre la critique structuraliste qui donne pour nuls les «effets de réel» et la critique sociologique et féministe qui prend en compte l'insertion du texte dans le monde et plus particulièrement dans le féminin puisque le personnage principal est une femme, Celia Rosenberg. La responsabilité du ou de la critique sera donc questionnée, mais aussi la responsabilité de l'écrivaine.

\section{Le rapport au texte de Duras: Hiroshima mon amour, publié en 1960}

Il ressort nettement des lectures successives des deux textes, de celui d'Hiroshima et de la Constellation du Cygne, que, pour l'intrigue, Yolande Villemaire n'a rien inventé. Elle a seulement emprunté et «hypertrophié» - avec tout ce que l'hypertrophie suppose d'excessif et d'anormal - l'épisode qui a eu lieu à Nevers pendant la guerre, entre une jeune Française et un soldat allemand. Au moment de la Libération, le soldat est tué. La honte de la liaison pèse lourd sur la jeune fernme: elle est tondue en place publique, comme il en était autrefois de l'exposition ou du pilori pour la femme adultère. Les parents l'enferment dans la cave de la maison, puis dans la chambre, et de là elle s'en ira à Paris. C'est au cours de l'aventure fulgurante qu'elle vit des années plus tard avec un Japonais à Hiroshima où elle est venue tourner un film sur la Paix que l'histoire de Nevers refait surface.

Le film de Duras n'a suscité qu'admiration et fascination. L'episode de Nevers a été pleinement accepté par le public et la critique. Hiroshima mon amour a pu devenir un classique de la littérature amoureuse et le film tourné par Resnais, un chef-d'oeuvre du cinéma.

D'où vient alors notre gêne à la lecture de la Constellation du Cygne?

D'une part, dans Hiroshima, il s'agit d'une jeune fille dans la fraîcheur du premier amour et l'aventure demeurera, quoi qu'il advienne, le temps de grâce de l'initiation heureuse.

2 Marguerite Duras, Hiroshima mon amour, Paris, Gallimard, 1960. 
Du soldat allemand, nous saurons seulement qu'il est jeune, beau, attentif à la musique que la jeune fille joue le soir au piano et qu'il écoute de la rue. Dans son anonymat de militaire, on peut le supposer autant victime que bourreau.

D'autre part, dans la Constellation du Cygne, la femme n'a rien d'une ingénue. C'est une prostituée: Ce ne sera pas son premier soldat allemand est-il écrit en page 12. La prostitution est là: par les vêtements longuement décrits, par le paiement que reçoit la femme pour les activités auxquelles elle se livre avec l'homme.

Quant à l'Allemand, il est officier et fait partie des SS. Il y a eu choix et la responsabilité ne fait pas de doute: l'Allemand est entré de plain-pied dans le jeu du nazisme. À la fin du livre, on le retrouve occupant un poste important, à Auschwitz.

Autre emprunt à Duras: l'inceste. Dans Agatha, l'inceste entre le frère et la soeur, non consommé, ordonne toute l'oeuvre. Ici il y a eu inceste, mais Villemaire force la dose, et l'inceste a bel et bien eu lieu. Je cite: Comment il devient son tyran, son maître, son amant. Elle dit l'amour éperdu pour son frère Julian. Et la haine aussi. D'un même souffle (p. 42). Là non plus, Yolande Villemaire n'a rien inventé. Mais cette fois l'inceste n'est là que pour donner du piment à une aventure déjà épicée. Villemaire mise sur l'excès comme pour s'assurer que son roman ne passe pas inaperçu.

Mais le rapport à Duras dépasse de beaucoup l'emprunt de l'intrigue et du passé de la femme. Il est inscrit dans les mots mêmes de Villemaire qui joue sur les effets de mode du texte durassien:

- par les répétitions de mots qui sont le propre de l'écriture de Duras. Par exemple, le mot "gris» revient trois fois dans la page 11, cinq fois dans la page 39 , six fois dans la page 45 , deux fois dans la page 53 , (et je n'ai pas utilisé l'ordinateur pour débusquer les récurrences...), le «gris» étant l'élément médiateur d'où tout peut vaciller, vers le noir ou vers le blanc également très présents.

La répétition des prénoms et noms des héros, Celia Rosenberg et Karl-Heinz Hausen, toujours donnés in extenso rappellent de très près l'attention portée par Duras aux noms propres, la signifiance accordée aux noms, bien mise en évidence par l'étude de Marcelle Marini dans Territoires du féminin avec Marguerite Duras ${ }^{3}$, par Nicole Bourbonnais dans «la Traversée de la musique ou: il n'y a rien à dire» 4 et par Lacan dans l'attention qu'il a donnée au nom de Lol

3 Marcelle Marini, Territoires du féminin avec Marguerite Duras, Paris, Minuit, 1977.

4 Nicole Bourbonnais, «la Traversée de la musique ou: il n'y a rien à dire», Colloque de l'APFUCC, Winnipeg, 1986. 
V. Stein 5 . Or, une héroïne de la Constellation du Cygne se nomme LolaValérie.

La relation aux textes de Duras est consciente; elle n'est pas non plus interdite. Mais que vaut cette exploitation de textes antérieurs? Voilà la question. Le mimétisme est évident dans la construction syntactique. Les phrases sont courtes, d'une simplicité qu'on dirait appliquée tant elle paraît peu naturelle, par le fait qu'elle tranche nettement sur la forme narrative de la Vie en prose. Comme chez Duras, les phrases comportent des fragments de reprise. Par exemple: Elle se met à lui parler d'elle. D'elle et de lui. De son amour pour lui. De son désir de lui (p. 42).

Rien de comparable cependant à l'effet incantatoire des formules archiconnues d'Hiroshima: Non, tu n'as rien vu à Hiroshima ou à celle-ci: Tu me tues. / Tu me fais du bien tant de fois entendue et jusque dans un film pornographique!

Sans doute Barthes et Lacan nous ont-ils sensibilisé(e)s à limpact du nom propre, non sans raison d'ailleurs et pour notre plus grande jouissance. Mais ici, dans la Constellation du Cygne, la répétition des noms et prénoms en partie justifiable du fait que les héros ne peuvent communiquer par le langage, chacun étant dans l'ignorance de la langue de l'autre, tourne à la monotonie et au tic, au besoin de combler artificiellement un manque. On s'accroche aux nom et prénom comme à une bouée, pour masquer le vide.

Autre emprunt à Hiroshima. La «gifle»6 que le Japonais envoie à la femme se multiplie chez Villemaire en «gifles» échangées à plusieurs reprises.

Le rapport à Duras s'étend du contenu à la forme. Mais alors que l'épisode de Nevers ne venait dans Hiroshima que comme dissociation, contrepoint et articulation du privé au politique, ici, la relation amoureuse occupe tout l'espace, devient le seul motif du roman. On mesure par là l'enflure que Villemaire lui a fait subir et en même temps on saisit combien le propos perd de sa dimension, puisque chez Duras, l'événement même d'Hiroshima était tel qu'il était constamment replacé dans l'ordre de l'indicible.

\section{Le réalisme du roman}

Le roman se donne pour réaliste par la minutie avec laquelle sont traitées dans les premières pages les scènes sexuelles. On y trouve le soin, la complaisance apportées dans les récits pornographiques:

5 Jacques Lacan, «Hommage fait à Marguerite Duras, du Ravissement de Lol V. Stein», dans Cahiers M. Renaud-J.L. Barrault, 52, décembre 1965.

6 Op. cit., p. 100. 
Il la rattrape, la retourne sur le ventre, remonte sa robe, arrache sa culotte et la pénètre brusquement par derrière. Celia Rosenberg, à quatre pattes, rit toujours. Mais bientôt, elle cesse de rire. L'homme la pénètre avec une certaine violence. Il parle, en allemand. Il hurle. Celia Rosenberg prend peur. Elle dit: "Arrête." Il s'arrête. Se penche sur sa nuque, embrasse sa nuque, caresse ses seins. Remue doucement un doigt sur son clitoris. Il vrille son pénis avec lenteur dans son vagin. Il se durcit davantage, s'immobilise. Il vient en silence, s'affale sur le dos de Celia Rosenberg qui s'écroule sur la moquette café-au-lait.

Il se relève, retourne délicatement Celia Rosenberg et, maniant agilement sa langue dans sa vulve, il la fait jouir avec sa bouche. Celia Rosenberg tente de se dégager mais il la retient, plaque de nouveau sa bouche sur son sexe. Celia Rosenberg explose encore tandis que le soldat allemand boit sa cyprine et la conduit d un autre orgasme (p. 3233).

Le voyeur est satisfait, la peinture est détaillée. Remarquons toutefois que la violence dans la pénétration, que le pénis toujours dur, appartiennent au registre de la pornographie, qu'ainsi s'établit un lien entre le désir pour la femme, la recherche des origines et la mort. La violence est signe du besoin de profanation de la mère sans doute, au pis-aller des autres femmes. Du désir que la femme inspire au nazi, celui-ci se vengera d'ailleurs en l'envoyant à la mort. $\mathrm{Ne}$ sommes-nous pas en pleine pornographie et dans le mythe le plus ignominieux de tous, celui de la complicité des victimes dans leur propre destruction ${ }^{8}$ comme le dit Nancy·Huston?

Mais le réalisme est là tout de même, dans la description attentive des dessous de la prostituée:

Elle passe un soutien-gorge de soie noire, des bas de soie noire qu'elle attache avec des jarretelles élastiques agrémentées de rubans blancs. Elle enfile une culotte de soie noire, un jupon de dentelle noire. Elle se dirige vers la table de nuit, y prend un flacon de parfum, en dépose quelques gouttes au creux de ses coudes et de sa nuque, revient derrière le paravent pour y chausser ses délicats escarpins en crocodile (p. 28).

Si j'ai substitué au mot «érotisme», celui de «pornographie», ce n'est pas anodin. C'est que l'érotisme, dans un mouvement de spirale, suppose une part de langage: le geste appelle la parole, les mots dérivent en contacts de plus en plus intimes, paroles et mouvements se mêlent, s'interpénétrent et ainsi de suite. Or, ici de langage, il n'y a pas. L'échange est impossible. N'existent que la voix, son timbre, ses inflexions, soit ce qui relève seulement du corps dans le langage oral.

7 La syntaxe ici n'y trouve pas son compte. Ce «il» renvoie à l'homme; quand au «il» suivant, il renvoie au pénis.

8 Nancy Huston, Mosaïque de la pornographle, Paris, Denoël/Gonthier, 1982, p. 139. 
Sur d'autres points, les invraisemblances sont nombreuses. J'en signale quelques-unes: les prostituées ne citent généralement pas Baudelaire (p. 53) et ne fréquentent pas davantage les salons comme cela se produit dans ce roman. Les salons parisiens ne s'ouvrent pas si facilement. Aussi l'intrusion de Celia Rosenberg paraît plutôt cocasse dans une soirée mondaine qui regorge de Juifs et de résistants, et aussi d'Allemands (p. 93)!

Les récits d'ex-prostituées qui se sont multipliés ces dernières années (dont on trouvera une mini-liste en note), tout autant que la lamentable histoire des prostituées de Grenoble, nous ont bien convaincu(e)s que, si la prostituée ne se sent pas toujours dans un ghetto comme ce fut le cas de Jeanne Cordelier, du moins, elle est marginale et comme le dit Nancy Huston isolée de façon radicale et souvent irrévocable 9 . L'étude de Nancy Huston, Mosaïque de la pornographie, est tout à fait éclairante pour la lecture de la Constellation du Cygne du fait qu'elle est en grande partie fondée sur le récit d'une prostituée, Marie-Thérèse10, qui a justement pratiqué le métier pendant la guerre, qu'elle a suivi un Allemand dans son pays où elle a séjourné de 1941 à 1945, d'où elle est revenue, pour «travailler» avec les Américains et les Noirs américains. Ce récit n'aurait-il pas inspiré celui de Yolande Villemaire?

Autre invraisemblance, majeure celle-ci: la liaison d'une Juive et d'un nazi. Il faut être naïve pour supposer qu'une Juive ait pu être d'un aveuglement absolument remarquable 11 selon les propres termes qu'emploie Yolande Villemaire dans son interview. Que des rapports aient eu lieu entre Juives et nazis dans les camps relève d'une autre histoire, celle des camps. Mais en 1940, une prostituée juive à Paris, ne pouvait qu'avoir envie de fuir à la seule vue d'un soldat allemand. Sans doute le vers de Boileau: le vrai peut quelquefois n'être pas vraisemblable peut justifier l'intrigue villemairienne, mais le comportement exceptionnel de la femme cadre bien mal avec l'état d'esprit des Juifs pendant la dernière guerre. Tout Juif connaît un minimum d'histoire de son peuple: les exactions, les vexations, les pogroms. Tout Juif sait qu'à un moment ou à un autre, le volcan antisémite peut produire une nouvelle éruption. Aussi l'attirance qu'exerce le nazi sur la prostituée me semble relever davantage des emprunts au littéraire ou au cinéma qu'à la réalité. Par exemple au film de Liliana Cavani: Portier de nuit (1973), fondé sur une relation sado-masochiste. La liaison paraît de la plus haute fantaisie. D'autant plus que peu de prostituées sont issues de la collectivité juive. L'aventure de la Juive avec le nazi soutient mal la confrontation avec des documents historiques sérieux, avec le film de Jacques Lanzmann: Shoah, par exemple.

$9 \quad$ Ibid., p. 176.

10 Marie-Thérèse, Vie d'une prostituée, 1950, Histoire d'une prostituée, Paris, Gonthier, 1964.

11 Yolande Villemaire, Entrevue avec Lucie Robert, op. cit., p. 397. 
Des anachronismes de moindre importance ne font que souligner la légèreté avec laquelle Yolande Villemaire a ancré son récit durant la guerre. En page 12, il est question de l'oeil charbonneux de Celia Rosenberg, à la page 29: [elle] dessine minutieusement ses yeux en noir. À cette époque les femmes utilisaient du rimmel, soit du fard pour les cils, mais les modes de l'oeil ourlé de noir, à l'égyptienne, et de l'oeil de biche sont postérieures au temps de la guerre. Pour s'en assurer, il suffit de revoir des films de l'époque. Ces détails ont de l'importance dans la mesure où l'auteure ayant voulu faire un roman réaliste et accrocheur - les scènes sexuelles sont là à cette fin -, la précision et l'abondance des détails font défaut. Exemple de légèreté encore: Celia Rosenberg ne connaît pas un traître mot d'allemand (p. 40, mais dans le Magazine littéraire, dans une entrevue, Yolande Villemaire dit: en tant qu'Alsacienne elle parle français et allemand [no d'octobre 86, p. 109]).

Question cruciale à propos de ce roman? De quel lieu le lira-t-on? Selon l'optique structuraliste, à la façon dont, dans les années 70 , ont été lus les textes de Sade, Bataille, Klossowski, traités en objets autonomes, position qui faisait dire à Barthes: l'auteur (matériel) d'un récit ne peut se confondre en rien avec le narrateur de ce récit ${ }^{12}$, qui parle (dans le récit) n'est pas qui écrit (dans la vie) et qui écrit n'est pas qui est, et de citer en note, Lacan à la rescousse: Le sujet dont je parle quand je parle est-il le même que celui qui parle13? Traiterons-nous Celia Rosenberg seulement comme être de papier ${ }^{14}$ selon les mots de Barthes?

Il semble bien que les limites de ce genre de critique ont été clairement établies depuis plusieurs années, dans la revue Texte de Toronto, en particulier (no 1, 1982).

Sans doute n'y a-t-il pas coalescence entre l'auteur et le narrateur, mais une certaine ambiguîté demeure, bien relevée par cette appréciation de Nicole Mozet:

Le roman réaliste est un genre hypocrite, fonctionnant sur le mode de la tentation. Exercice dangereux pour celui qui en prend la responsabilité et qui risque a tout instant de se voir mis en accusation. Dans tout lecteur, il y a un censeur en puissance. D'ou la nécessité de pouvoir refuser une identification mortelle entre le je de l'écriture et celui de la signature, alors que toute la nouvelle pratique littéraire, aussi bien dans ses modes de production que dans la façon dont elle est reçue, tend à hausser cette identification au rang d'évidence.

La question de l'énonciation y compris le sexe des auteurs se pose.

12 Roland Barthes, «Introduction à l'analyse structurale des récits», Communications, no 8, 1966, p. 25.

13 Roland Barthes, op. cit., p. 26 et note 1, même page.

14 Ibid., p. 25. 
Pour Nicole Mozet, aucun doute, l'on reste prisonnier de ce que l'on écrit 15. À noter aussi sur la couverture du livre Y. Villemaire en Celia Rosenberg. Selon l'auteure, elle aurait été C. Rosenberg dans une vie antérieure.

En effet le «sexe des auteurs» a affaire avec l'écriture si on ne croit pas à une écriture qui transcenderait la différence des sexes. Je suis tellement d'accord avec cette position que, selon ce que j'ai relevé dans Mosaique de la pornographie, je pourrais classer le livre de Yolande Villemaire (et je cite) dans le versant rose (appréciez cette épithète rose appliquée à Villemaire) de la prostitution. Nancy Huston parle alors de ces prostituées littéraires qui sont par nature jouisseuses, insatiables (...) qui font l'amour par inclination plutôt que par nécessitét 16 . N'estce pas à cette espèce de prostituées qu'appartiendrait Celia Rosenberg?

En fait, il est assez facile de montrer que ce livre qui se love si bien dans les schémas masculins est, par le fait même, anti-féministe. Celia Rosenberg n'a pas une once de lucidité, comme le reconnaît l'auteure elle-même dans l'entretien. Celia Rosenberg n'existe que par le corps, par cela même par quoi les productions pornographiques tiennent la femme dans un état subalterne. De cette femme, il n'y a strictement rien à attendre. Elle est l'être le plus aliéné qui soit, triplement en tant que femme, en tant que prostituée, en tant que Juive durant la guerre. Elle est si attentive aux seules pulsions de son corps que lorsque ses yeux - enfin se dessillent, il est si tard que cela n'a plus d'importance.

Si la phrase le privé est politique est devenue le leitmotiv de la pensée féministe, si la réflexion des femmes est inscrite jusque dans leur privé, que devient Celia Rosenberg en regard de ces enjeux?

Elle est hors champ, du fait qu'elle relève d'une vision édulcorée de l'Histoire. Dès les premières pages, l'ésotérisme intervient sous les traits de la magicienne à la peau verte qui, à intervalles réguliers, représente les infiltrations venues d'un autre monde. Des phrases comme celle-ci: On ne dirait pas que c'est la guerre. Mais ça se sent (p. 55), par leur vacuité, ne renvoient à rien de précis, ne veulent rien dire pour qui a quelque expérience de l'occupation nazie ou des régimes fascistes. Le roman paraît ancré dans un terrain si vague que les vertus de la métempsycose viennent comme un deus ex machina mettre tout en ordre, faire en sorte que les forces du Bien l'emportent sur les forces du Mal.

Mais de quelle aide nous sont ces mancies, pour qu'en fin de compte, Celia Rosenberg s'en tire en galopant sur un cheval bleu dans l'au-delà, qu'elle revienne partout sur la terre, dans d'autres corps, dans les corps de ceux et celles qui sont encore vivants, et dans d'autres galaxies (p. 178)? La réincarnation ne fait pas oublier ce qui se passe au Chili, l'abcès purulent de l'Afghanistan, le terrorisme à Paris. Le danger nucléaire dont parle Yolande Villemaire existe aussi, mais plus

15 Nicole Mozet, «la Femme-auteur comme symptôme», dans Femmes et institutions littéraires, Cahiers de recherches, S.T.D./Paris VII, no 13, 1984.

16 Nancy Huston, op. cit., p. 27. 
près de nous, d'autres tortures, d'autres meurtres qui ne sont pas à venir, mais actuels.

L'Histoire avec un grand $\mathrm{H}$ a été assimilée dans ce roman à l'histoire d'amour, alors que d'un romancier, c'est le contraire qu'on attend, soit que la petite histoire soit intégrée à la grande, parce que c'est Elle qui mène le jeu et non l'inverse. Les Juifs n'ont pas eu le choix de mourir dans leur lit ou à Auschwitz. Cela, Yolande Villemaire ne l'a pas pris en compte.

$\mathrm{Si}$, en tant que lectrice, je prends une lourde responsabilité - en regard de la consécration dont jouit Yolande Villemaire au Québec, c'est aussi que je peux en appeler à sa responsabilité d'écrivaine. Je ne conteste pas sa croyance dans les vies antérieures. Je dis seulement que le sujet ne m'intéresse pas, ayant déjà fort à faire avec la vie d'ici-bas. Mais je n'accepte pas que cette croyance serve de panacée, d'échappatoire. Et je ne puis que m'élever contre ces paroles de Villemaire dans l'entretien accordé:

Près d'un feu de camp, chez des amis, j'ai rencontré un vieux Belge qui avait été membre des SS noirs et qui me disait qu'il avait été d'accord avec l'holocauste parce que les Juifs étaient une race inférieure. On était en août 1983 au Québec. Il disait ça sans vergogne. (...)

J'ai commencé d avoir mal d̀ la jambe. J'ai passé une semaine à l'hôpital et j'ai traîné pendant un mois et demi.

Maintenant je peux voir que cette histoire a libéré l'engramme. (...)

J'ai vécu le roman. J'ai retrouvé des parcelles, des poches d'émotion. En les traversant, $j$ 'ai atteint une position mystique, religieuse, qui est reliée à la mort: j'ai atteint le pardon. En écrivant la Constellation du Cygne, je me suis trouvée à pardonner à Hitler. C'est une position inconfortable. Je ne nie pas la souffrance, mais je pense que les erreurs du passé sont là pour nous permettre de ne pas les reproduire 17 .

Qui est Yolande Villemaire pour pardonner ainsi à Hitler et au nazisme?

Et quand Yolande Villemaire en appelle à l'hologramme comme image qui traduit l'unité, ce n'est pas sans légèreté puisqu'elle «avoue» (...): je ne sais pas ce qu'est exactement un hologramme.

Ainsi, «pardonner à Hitler», incarnation du nazisme, s'en remettre à l'hologramme, tout cela n'est pas très sérieux. Du moins pour le domaine de l'immanence. À moins que nous ne fassions comme elle et vivions dans l'espoir que la réincarnation nous sauve tous, un jour, peut-être!

Quant à l'existence même d'une écriture dans ce roman, elle aussi fait problème et l'article de Claude Sabourin, paru dans le numéro de Voix \& images dont il a été question, apporte un éclairage dur sur le sujet. Encore là, lacis d'interrogations...

17 Yolande Villemaire, Entrevue, op. cit., p. 397. 
Livres écrits par des prostituées:

DE CONINCK, Barbara et Christine, la Partagée, Paris, Minuit, 1977.

JAGET, Claude, Une vie de putain, Paris, Presses d'aujourd'hui, 1975.

LOVELACE, Linda, Ordeal, New York, Berkeley Books, 1981.

CORDELIER, Jeanne, la Dérobade, Paris, Hachette, 1976.

AZIZ, Geneviève, les Chambres closes, Paris, Stock, 1980.

HENNIG, Jean-Luc, Grisélidis, courtisane. Entretiens, Paris, Albin Michel, 1981.

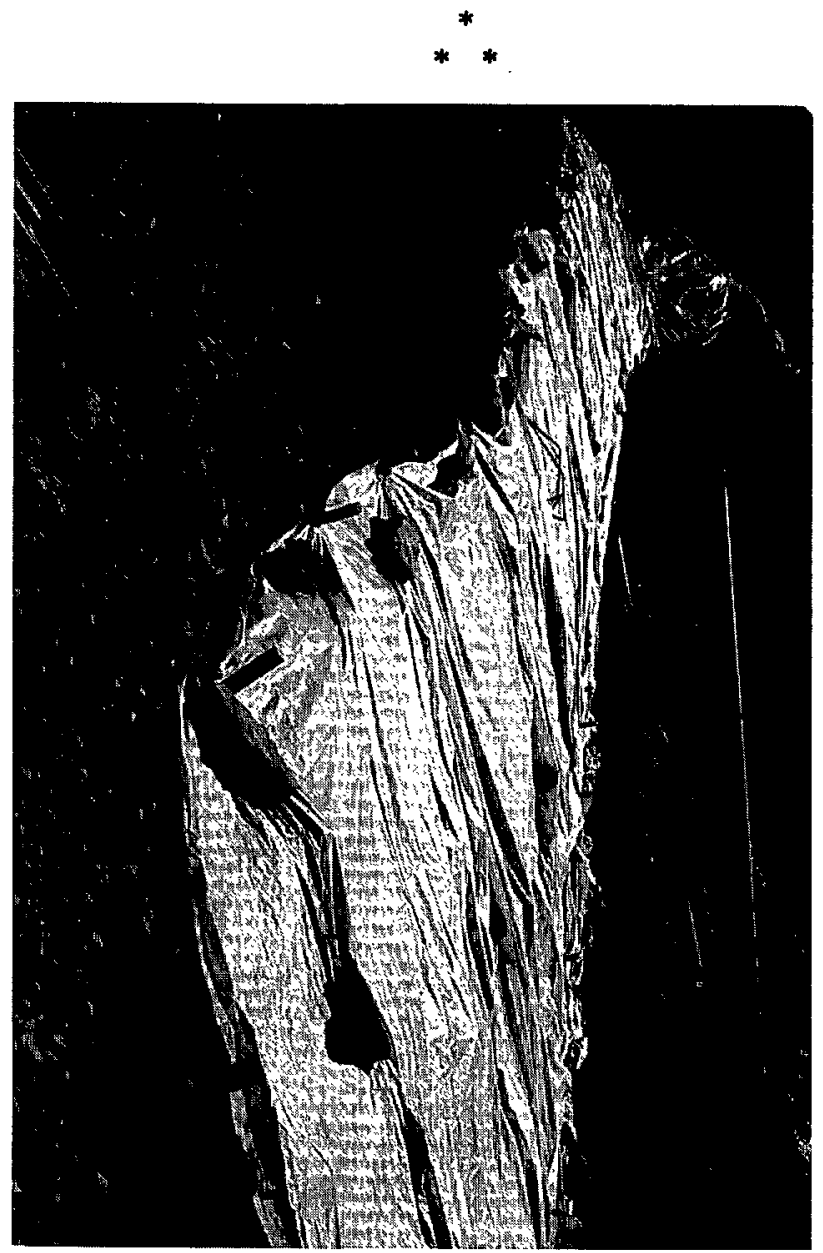

